\title{
Front Matter: Volume 10019
}

, "Front Matter: Volume 10019," Proc. SPIE 10019, Optoelectronic Devices and Integration VI, 1001901 (27 January 2017); doi: 10.1117/12.2266314

SPIE. Event: SPIE/COS Photonics Asia, 2016, Beijing, China 


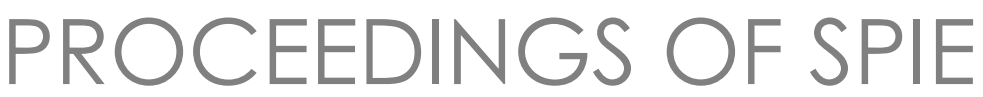

\title{
Optoelectronic Devices and Integration VI
}

\author{
Xuping Zhang \\ Baojun Li \\ Changyuan Yu \\ Editors
}

\section{2-14 October 2016 \\ Beijing, China}

Sponsored by

SPIE

COS-Chinese Optical Society

Cooperating Organizations

Tsinghua University (China) - Peking University (China) • University of Science and Technology of China (China) •

Zhejiang University (China) • Tianjin University (China) • Beijing Institute of Technology (China) • Beijing University of Posts and Telecommunications (China) • Nankai University (China) • Changchun University of Science and Technology (China) • University of Shanghai for Science and Technology (China) • Capital Normal University (China) • Huazhong University of Science and Technology (China) • Beijing Jiaotong University (China) - Shanghai Institute of Optics and Fine Mechanics (China) • Changchun Institute of Optics and Fine Mechanics (China) • Institute of Semiconductors (China) • Institute of Optics and Electronics (China) • Institute of Physics (China) - Shanghai Institute of Technical Physics (China) - China Instrument and Control Society (China) • Optoelectronics Technology Committee, COS (China) • SPIE National Committee in China (China) • Optical Society of Japan (Japan) • Optical Society of Korea (Korea, Republic of) - The Australian Optical Society (Australia) - Optics and Photonics Society of Singapore (Singapore) • European Optical Society

\section{Supporting Organizations}

CAST_China Association for Science and Technology (China)

NSFC-National Nature Science Foundation (China)

Published by

SPIE

\section{Volume 10019}


The papers in this volume were part of the technical conference cited on the cover and title page. Papers were selected and subject to review by the editors and conference program committee. Some conference presentations may not be available for publication. Additional papers and presentation recordings may be available online in the SPIE Digital Library at SPIEDigitallibrary.org.

The papers reflect the work and thoughts of the authors and are published herein as submitted. The publisher is not responsible for the validity of the information or for any outcomes resulting from reliance thereon.

Please use the following format to cite material from these proceedings:

Author(s), "Title of Paper," in Optoelectronic Devices and Integration VI, edited by Xuping Zhang, Baojun Li, Changyuan Yu, Proceedings of SPIE Vol. 10019 (SPIE, Bellingham, WA, 2016) Six-digit Article CID Number.

ISSN: 0277-786X

ISSN: 1996-756X (electronic)

ISBN: 9781510604575

ISBN: 9781510604582 (electronic)

Published by

SPIE

P.O. Box 10, Bellingham, Washington 98227-0010 USA

Telephone +1 3606763290 (Pacific Time) · Fax +1 3606471445

SPIE.org

Copyright (C) 2016, Society of Photo-Optical Instrumentation Engineers.

Copying of material in this book for internal or personal use, or for the internal or personal use of specific clients, beyond the fair use provisions granted by the U.S. Copyright Law is authorized by SPIE subject to payment of copying fees. The Transactional Reporting Service base fee for this volume is $\$ 18.00$ per article (or portion thereof), which should be paid directly to the Copyright Clearance Center (CCC), 222 Rosewood Drive, Danvers, MA 01923. Payment may also be made electronically through CCC Online at copyright.com. Other copying for republication, resale, advertising or promotion, or any form of systematic or multiple reproduction of any material in this book is prohibited except with permission in writing from the publisher. The CCC fee code is 0277-786X/16/\$18.00.

Printed in the United States of America.

Publication of record for individual papers is online in the SPIE Digital Library.

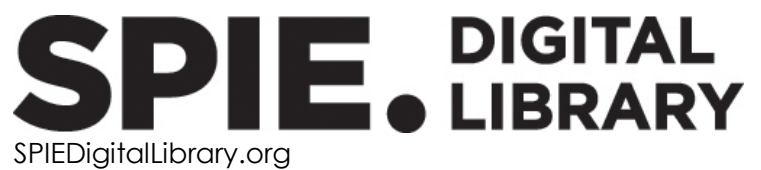

Paper Numbering: Proceedings of SPIE follow an e-First publication model. A unique citation identifier (CID) number is assigned to each article at the time of publication. Utilization of CIDs allows articles to be fully citable as soon as they are published online, and connects the same identifier to all online and print versions of the publication. SPIE uses a seven-digit CID article numbering system structured as follows:

- The first five digits correspond to the SPIE volume number.

- The last two digits indicate publication order within the volume using a Base 36 numbering system employing both numerals and letters. These two-number sets start with 00, 01, 02, 03, 04, $05,06,07,08,09,0 A, 0 B \ldots$.. 0Z, followed by 10-1Z, 20-2Z, etc. The CID Number appears on each page of the manuscript. 


\title{
Contents
}

\author{
vii Authors \\ ix Symposium Committees \\ xi Conference Committee
}

SESSION 1 INTEGRATED OPTICS AND PHOTONIC INTEGRATED CIRCUITS

1001905 Silicon nitride grating waveguide based directional coupler [10019-5]

SESSION 2 DEVICES FOR PHOTONIC APPLICATIONS I

$1001907980 \mathrm{~nm}$ tapered lasers with photonic crystal structure for low vertical divergence [10019-7]

1001908 Tunable multi-channel dropping filters based on double-waveguide parallel-coupled microring resonators [10019-8]

1001909 The realization of optical switching generated from the combination of $\mathrm{Ag} / \mathbf{a}-\mathrm{Si} / \mathrm{p}-\mathrm{Si}$ memristor and silicon waveguide [10019-9]

10019 OA High efficiency single transverse mode photonic band crystal lasers with low vertical divergence [10019-10]

10019 OB Preparation and evaluation of perovskite solar cells in the absolute atmospheric environment [10019-19]

\section{SESSION 3 EMERGING OPTOELECTRONIC APPLICATIONS}

10019 OC Investigation of advanced pre- and post-equalization schemes in high-order CAP modulation based high-speed indoor VLC transmission system (Invited Paper) [10019-12]

10019 OE Optical implementation of neural learning algorithms based on cross-gain modulation in a semiconductor optical amplifier [10019-14]

10019 OF Detection of atmospheric boundary layer height in the plum rain season over Hangzhou area with three-dimensional scanning polarized lidar [10019-15]

10019 OG Design of real-time and high-precision photoelectric autocollimator [10019-16] 
$10019 \mathrm{OH}$ Development of next-generation nanolithography methods to break the optical diffraction limit (Invited Paper) [10019-17]

10019 OK Gain-switched thulium-doped fiber laser with ultra-wide tuning range [10019-20]

10019 OL Er-doped fiber based Mach-Zehnder interferometer for simultaneous strain and temperature measurement [10019-53]

SESSION 5 OPTICAL FIBERS

10019 ON Mode evolution in polarization maintain few mode fibers and applications in modedivision-multiplexing systems (Invited Paper) [10019-22]

10019 OP Experimental measurement of effective refractive index difference for few mode polarization maintaining fibers using $S^{2}$ method [10019-24]

$100190 Q \quad$ Uniform flat-top interleaver consisting of a two-stage cascaded Mach-Zehnder interferometer [10019-25]

10019 OR A distributed optical fiber sensing system for dynamic strain measurement based on artificial reflector [10019-26]

10019 OS Improved hybrid polymer/PbS quantum dot infrared phototransistors incorporating singlelayer graphene [10019-27]

10019 OT A calibration method for photon counters using a customized standard light source [10019-28]

10019 OU Coupling between fiber-optic microring and lithium niobate microwaveguide chip towards photonic interlink devices [10019-29]

10019 OV Investigation on a novel fiber-optic sensor based on up-taper-core-offset-up-taper structure [10019-52]

\section{SESSION 6 PHOTONIC MATERIALS AND DEVICES/DETECTORS I}

10019 OW A simple and low cost method to fabricate well controllable silicon nanowires based on electrostatic adsorption of PS nanospheres [10019-30]

\section{SESSION 7 PHOTONIC MATERIALS AND DEVICES/DETECTORS II}

1001911 Performance optimization of Pnp InGaAs/InP heterojunction phototransistors [10019-36]

1001912 On-chip integration for in-plane video transmission using visible light [10019-37] 
1001913 Optical characteristic analysis of optical fiber near-field and far-field [10019-38]

1001914 Investigation on optical and acoustic fields of stimulated Brillouin scattering in $\mathrm{As}_{2} \mathrm{~S}_{3}$ suspended-core optical fibers [10019-39]

1001915 A short-range optical wireless transmission method based on LED [10019-40]

1001917 Directional emission micro-cavity lasers with different device structures [10019-42]

1001918 The research of multi-alkali vacuum photodiode on heating and illuminating [10019-43]

1001919 Chromatic dispersion and polarization mode dispersion insensitive optical signal to noise ratio monitoring based on electronic variable optical attenuators and optical bandpass filter [10019-44]

$100191 \mathrm{~A} \quad$ All-optical quantization by slicing supercontinuum in a Ge ${ }_{11.5} \mathrm{As}_{24} \mathrm{Se}_{64.5}$ rib waveguide [10019-45]

10019 1B High-performance transparent film heater with an embedded Ni metal-mesh based on selected metal electrodeposition process [10019-46]

10019 1C Multimode fiber focusing lens based on plasmonic structures [10019-47]

10019 1D High precision long-term stable fiber-based optical synchronization system [10019-48]

10019 1E A four-port vertical-coupling optical interface based on two-dimensional grating coupler [10019-49]

10019 1F RF spectral analysis for characterisation of mode-locked regimes in fibre lasers [10019-50]

$100191 \mathrm{G}$ Solar cells based on InP/GaP/Si structure [10019-51] 
Proc. of SPIE Vol. $100191001901-6$

Downloaded From: https://www.spiedigitallibrary.org/conference-proceedings-of-spie on 25 Apr 2023 Terms of Use: https://www.spiedigitallibrary.org/terms-of-use 


\section{Authors}

Numbers in the index correspond to the last two digits of the seven-digit citation identifier (CID) article numbering system used in Proceedings of SPIE. The first five digits reflect the volume number. Base 36 numbering is employed for the last two digits and indicates the order of articles within the volume. Numbers start with $00,01,02,03,04,05,06,07,08,09,0 A, 0 B . .0 Z$, followed by 10-1Z, 20-2Z, etc.

Akimoto, Ryoichi, 05

Bai, Jian, OF

Cao, Mingxuan, os

Chang, Junde, 19

Che, Yongli, OS

Chen, Ailin, 15

Chen, Hongda, $1 \mathrm{E}$

Chen, Jun, 11

Chen, Li, 14

Chen, LinSen, 1B

Chen, Xiangcai, 14

Chen, Xiangdong, 14

Chen, Yang, OA

Chen, Zhe, OU

Chen, Zhi, OB

Cheng, Chuantong, $1 \mathrm{E}$

Cheng, X., OK

Cheng, Zhongtao, OF

Chi, Nan, OC

Dang, Hongmei, OB

Ding, $\mathrm{Li}, \mathrm{OH}$

Ding, Xin, OS

El-Zohary, Salah E., OW

Fan, Wei, 1D

Feng, Jijun, 05

Feng, Yuan, 17

Fu, Rongguo, 18

Gao, Tianxi, $1 \mathrm{E}$

Gao, Weiqing, 14

Gao, Xumin, 12

Gao, Yingming, 15

Gu, Huarong, OT

Guan, Chunying, 1C

Guang, Heyuan, OU

Guo, Anran, 09

Guo, Guohui, 09

Guo, Wenting, OP

Hao, Yong-qin, 17

Haraguchi, Masanobu, OW

Hou, J., OK

Hou, Jiayin, OG

$\mathrm{Hu}$, Jigang, 14

$\mathrm{Hu}$, Xiaochuan, $1 \mathrm{E}$

Huang, Beiju, $1 E$

Huang, Wenbin, 1B

Iguchi, Yusuke, OW

Ivanenko, Alexey V., IF

Jiang, Yadong, 09

Jiang, Youen, ID
Kang, Shuai, 1A

Kang, Zhe, IA

Kobłsev, Sergey M., IF

Kokhanovskiy, Alexey, $1 \mathrm{~F}$

Kvitsiani, O., 1G

Laperashvil, D., $1 G$

Laperashvili, T., $1 \mathrm{G}$

Le, Yansi, OE

Li, Anyuan, 05

Li, Dongyang, 09

Li, Hui, 08, 0Q

Li, Hui, 17

Li, Lianhuang, 13

Li, Lunhua, OA

Li, Peng, 17

Li, Ping, 15

Li, Qiang, OE

Li, Rao, 1D

Li, Wei, 09

Li, Wei, ON, OP

Li, Xue, 14

Li, Yan, ON, OP

Li, Yanting, OR

Li, Yuanhang, 12

Li, Yurong, 1D

Li, Z., OK

Lin, Peihua, 13

Lin, Shulang, OT

Lin, Yuzhe, OA

Liu, Anjin, OA

Liv, Bo, 19

Liu, Chong, OF

Liu, Dong, OF

Liu, Yanhua, 1B

Liu, Yanyang, OF

Liu, Yun, 07, 0A

Liu, Z., OK

Liu, Zhijian, ON, OP

Lu, Huihui, OU

Lu, Yongjiao, $1 \mathrm{C}$

Luo, Huiwen, $\mathrm{OH}$

Luo, Jing, OF

Ma, Xiaolong, 07

Mei, Chao, $1 \mathrm{~A}$

Miao, Meiyuan, 15

Mikelashvili, $\vee ., 1 G$

Mo, Qi, ON, OP

$\mathrm{Ni}$, Chenquan, 14

Okamoto, Toshihiro, OW 
Qi, Aiyi, OA

Qiao, Wen, 1B

Qiao, Zhi, 1D

Qin, Jin, $\mathrm{OH}$

Qu, Hongwei, 07, 0A

Rao, Lan, 19

Sang, Xinzhu, IA

Sarvari, Hojjatollah, OB

Shan, Yuanyuan, OR

Shen, SU, 1B

Shen, Yibing, OF

Shi, Jian-wei, 17

Shi, Jinhui, 1C

Singh, Vijay, OB

Smirnov, Sergey $V$. ., IF

Song, Qinjian, 09

Song, Xiaojia, OE

Song, Xiaoxian, OS

SU, Huaiyin, $O Q$

Sun, Chonghui, OE

Sun, Zhenhong, OR

Tan, Qiaofeng, OT

Tang, Peijun, OF

Tian, Feng, 19

Tian, Qinghua, 19

Wang, Guiyuan, 18

Wang, Jia-bin, 17

Wang, Jianlong, OS

Wang, Kaiwei, OF

Wang, Kuiru, $1 \mathrm{~A}$

Wang, Kun, 18

Wang, Liang, $\mathrm{OH}$

Wang, Shanshan, OG

Wang, Xiaochao, 1D

Wang, Xiaohui, OB

Wang, Yanyan, IB

Wang, Yiguang, OC

Wang, Yongjin, 12

Wang, Yongjun, 19

Wang, Yuan, OU

Wang, Zhi, OE

Wei, Yifang, 18

Wu, Chongqing, OE

WU, Jian, ON, OP

Wu, Yongfeng, 08, 0Q

Xin, Xiangjun, 19

Xu, Peituo, OF

$X \cup$, Qiang, 14

Xu, Wei, OL, OV

$X U$, Yihang, IC

Yan, Binbin, 1A

Yan, Chang-ling, 17

Yang, Liu, 18

Yang, Yongchao, 12

Yang, Yongying, OF

Yao, Jianquan, OS

Ye, Yan, 1B

Yin, Xiaoli, 19

You, Shanhong, OL, OV

Yu, Changqiu, 08, OQ
Yu, Changyuan, OL, OV

Yu, Cheungchuen, OL

YU, Chongxiu, 1A

YU, Jianhui, OU

YU, YU, OS

Yuan, Jialei, 12

Yuan, Jinhui, $1 \mathrm{~A}$

Yuan, Libo, $1 \mathrm{C}$

Yuan, Ping, 08, 0Q

Yuan, Zijun, 14

Zeng, Heping, 05

Zeng, Xinglin, ON, OP

Zhan, Zhenlin, 13

Zhang, Chunyu, 08

Zhang, Haiting, OS

Zhang, Jian-jia, 17

Zhang, Lijia, 19

Zhang, Lin, 1E

Zhang, Na, OL, OV

Zhang, Qi, 19

Zhang, Wei, 14

Zhang, Xianting, $1 \mathrm{~A}$

Zhang, Xuping, OR

Zhang, Yanlei, 1A

Zhang, Yating, OS

Zhang, Yixin, OR

Zhang, Yundong, 08, OQ

Zhang, Yupeng, OF

Zhang, Zan, $1 \mathrm{E}$

Zhang, Zanyun, $1 \mathrm{E}$

Zhao, Pengchao, 07

Zhao, Shaoyu, OA

Zheng, Wanhua, 07, 0A

Zheng, Xiaojie, OU

Zhou, Siyu, OG

Zhou, SUXu, OU

Zhou, Xuyan, OA

Zhou, Yudi, OF

Zhou, Yun, 1B

Zhu, Min, 11

Zhu, Mingxing, 15

Zhu, Qiudong, 0G

Zhu, Zheng, $1 \mathrm{C}$

Zou, Nianyu, 15 


\title{
Symposium Committees
}

\author{
General Chairs \\ Robert Lieberman, SPIE President, Lumoptix, LLC (United States) \\ Guangcan Guo, Chinese Optical Society President, University of \\ Science and Technology of China (China) \\ General Co-chairs \\ Arthur Chiou, National Yang-Ming University (Taiwan, China) \\ Jianlin Cao, China Ministry of Science and Technology (China) \\ Junhao Chu, Shanghai Institute of Technical Physics (China) \\ Technical Program Chairs \\ Songlin Zhuang, University of Shanghai for Science and Technology \\ (China) \\ Xingde Li, Johns Hopkins University (United States) \\ Technical Program Co-chairs \\ Bingkun Zhou, Tsinghua University (China) \\ Qiming Wang, Institute of Semiconductors (China) \\ Tianchu Li, National Institute of Metrology (China) \\ Wei Huang, Nanjing University of Technology (China) \\ Ying Gu, PLA General Hospital (China) \\ Huilin Jiang, Changchun University of Science and Technology \\ (China) \\ Local Organizing Committee Chair \\ Qihuang Gong, Peking University (China)
}


Local Organizing Committee Co-chairs

Xu Liu, Zhejiang University (China)

Daoyin Yu, Tianjin University (China)

Guoqiang Ni, Beijing Institute of Technology (China)

Shusen Xie, Fujian Normal University (China)

Xiaomin Ren, Beijing University of Posts and Telecommunications

(China)

General Secretary

Yan Li, Chinese Optical Society/Peking University (China)

Local Organizing Committee

Zhiping Zhou, Peking University (China)

Changhe Zhou, Shanghai Institute of Optics and Fine Mechanics, CAS

(China)

Qingming Luo, Huazhong University of Science and Technology

(China)

Chongxiu Yu, Beijing University of Posts and Telecommunication

(China)

Hongda Chen, Institute of Semiconductors (China)

Yongtian Wang, Beijing Institute of Technology (China)

Yiping Cui, Southeast University (China)

Xuping Zhang, Nanjing University (China)

Feijun Song, Daheng Corporation (China)

Cunlin Zhang, Capital Normal University (China)

Yanting Lu, Nanjing University (China)

Yuejin Zhao, Beijing Institute of Technology (China)

Chunqing Gao, Beijing Institute of Technology (China)

Tiegen Liu, Tianjin University (China)

Xiaocong Yuan, Nankai University (China)

Weimin Chen, Chongqing University (China)

Zhongwei Fan, Academy of Optoelectronics (China)

Hanyi Zhang, Tsinghua University (China)

Lan Wu, Zhejiang University (China)

Yongsheng Zhang, University of Science and Technology of China (China)

Hong Yang, Peking University (China)

Xiaoying Li, Tianjin University (China)

Wei Xiong, Chinese Optical Society (China) 


\title{
Conference Committee
}

\author{
Symposium Chairs
}

Robert A. Lieberman, Lumoptix, LLC (United States)

Wei Xiong, Chinese Optical Society (China)

Conference Chairs

Xuping Zhang, Nanjing University (China)

Baojun Li, Sun Yat-Sen University (China)

Changyuan Yu, National University of Singapore (Singapore)

Conference Program Committee

Dayan Ban, University of Waterloo (Canada)

Zhongping Chen, Beckman Laser Institute and Medical Clinic (United States)

Ho-Pui A. Ho, The Chinese University of Hong Kong

(Hong Kong, China)

Jan Ingenhoff, Ionexphotonics Inc. (Canada)

Zhong-cheng Liang, Nanjing University of Posts and Telecommunications (China)

Xuejun Lu, University of Massachusetts Lowell (United States)

Hai Ming, University of Science and Technology of China (China)

Bikash Nakarmi, Korea Advanced Institute of Science and Technology (Korea, Republic of)

Gang-Ding Peng, The University of New South Wales (Australia)

Yuan Shi, Agilecom Photonic Solutions Inc. (United States)

Yuejiang Song, Nanjing University (China)

Anna K. Swan, Boston University (United States)

Frank Vollmer, Max-Planck-Institut für die Physik des Lichts (Germany)

Guanghui Wang, The Chinese University of Hong Kong

(Hong Kong, China)

Daniel M. Wasserman, University of Illinois at Urbana-Champaign

(United States)

Lixin Xu, University of Science and Technology of China (China)

Session Chairs

1 Integrated Optics and Photonic Integrated Circuits

Changyuan Yu, National University of Singapore (Singapore)

2 Devices for Photonic Applications I

Wei Jiang, Nanjing University (China) 
3 Emerging Optoelectronic Applications

Baojun Li, Jinan University (China)

4 Devices for Photonic Applications II

Yiping Wang, Shenzhen University (China)

5 Optical Fibers

Changyuan Yu, National University of Singapore (Singapore)

6 Photonic Materials and Devices/Detectors I

Changyuan Yu, National University of Singapore (Singapore)

7 Photonic Materials and Devices/Detectors II

Changyuan Yu, National University of Singapore (Singapore) 